\title{
An Artificial Intelligence System to Predict Progression of Immune Dysfunction in Healthy Older Patients
}

\author{
E. M. Mahler, R. M. Schmidt, and V. I. Kvitash
}

In 1983, forty nine participants returning for their annual HEALTH WATCH evaluation were assessed for immune function status and served as healthy controls in an early study of "normal" reference values for $T_{4}$ and $T_{8}$ lymphocyte counts and $T_{4} / T_{8}$ lymphocyte ratios, B lymphocyte counts, and other laboratory tests for immune function then available. Balascopy, a computer-assisted pattern recognition system for detection, quantification and cognition of multiple imbalances among clinical laboratory and health behavioral parameters, facilitates identification of otherwise healthy individuals with evidence of early immune dysfunction. When the $T_{4}$ and $T_{8}$ immunology tests were combined with 7 health index behavioral subscores, and 24 routine clinical laboratory tests, we are able to obtain a bodyprint unique for each patient, providing a comprehensive health status profile specific for that individual. These bodyprints serve as tools for the evaluation of otherwise undetectable metabolic, immunological and behavioral imbalances in a given patient in an unprecedented individualized manner. We show here a longitudinal look at a balascopic evaluation of one patient who has evidence of progressive immune dysfunction with development of only mild clinical symptoms.

\section{MATERIALS AND METHODS}

Health Watch is a longitudinal prospective study of healthy aging through which physicians and other health care providers can use person-specific data to monitor health and to identify interventions, while researchers continue to assess aging effects and determinants of healthy aging. The study uses a computer-based monitoring system for tracking the biochemical, hematological, physiological and behavioral parameters $(\mathrm{n}=38)$ in men and women over their lifespan. This "person-specific" data has been studied in close to 3000 individuals for up to 23 years. Health Trend Charts summarize annual updates for biochemical $(n=17)$, hematological $(n=8)$ and physiological $(n=6)$ parameters. A Health Profile Index, derived from a questionnaire updated by each patient annually, provides 7 subscores for health status. The Health Watch approach allows the primary care physician to observe early trends of unhealthy behavioral or laboratory patterns prior to signs or symptoms of disease, thereby permitting the active practice of primary preventive medicine, in partnership with the individual patient. The clinical parameters assessed annually as well as the seven health status and health behaviors subscores are listed in Table 1.

Balascopy (Balance + Scope) is a computer assisted methodology developed for the analysis of relationships between multiple immunological, hematological, biochemical, physiological and behavioral parameters. Balascopy utilizes a pattern recognition system for detection, quantification and cognition of imbalances in the above relationships. Generated balascopic patterns help to predict the presence or illustrate the development of immunodeficiencies in otherwise healthy individuals at the routine clinical laboratory or physician level office. Longitudinal analysis of balascopic interpretations of patientspecific data provides for 
early, correct identification of progressive immune dysfunction and increased patient susceptibility to infectious diseases. Balascopic information, which is not available from any other existing modality, represents a new class of knowledge and opens up a new method for studying the mechanisms of disease and designing individualized therapeutic approaches. ${ }^{1-5}$

Table 1. Health Watch Clinical Parameters and Health Behavior Subscores

\begin{tabular}{ll}
\hline Hematology & \\
White blood cell count & Mean cell volume \\
Red blood cell count & Mean cell hemoglobin \\
Hemoglobin & Mean cell hemoglobin concentration \\
Hematocrit & Platelets
\end{tabular}

Blood serum chemistry

$\begin{array}{lll}\text { Glucose } & \text { Urea nitrogen } & \text { Aspartate aminotransferase } \\ \text { Creatinine } & \text { Uric acid } & \text { Gamma glutamyltransferase } \\ \text { Calcium } & \text { Phosphorus } & \text { Triglyceride } \\ \text { Total protein } & \text { Cholesterol } & \text { Lactic dehydrogenase } \\ \text { Thyroxine } & \text { Alkaline phosphatase } & \text { High density lipoprotein } \\ \text { Total cholesterol } & \text { Low density lipoprotein } & \end{array}$

Body physiology

Height Systolic blood pressure

Weight Diastolic blood pressure

Heart rate Percent body fat

Health status and health behavior subscores (examples of 1340 variables)

Nutrition: Caffeine use, fat and fiber intake, nutritional adequacy

Tobacco: Cigarette, cigar, pipe use

Alcohol: Wine, beer, hard liquor consumption

Exercise: Aerobic benefit, flexibility, endurance, frequency and duration of workout, balance

Medical events: Aspirin use, accidents, exposures, illness, surgery, medication use

General well-being: Number of headaches and colds, subjective health, sleep pattern, number of sick days taken

Psychological well-being: Work performance and satisfaction, stress levels at work and at home, financial stress, stressful life events (marriage, death of a friend, etc.) 
The charts and balascopic interpretations presented here represent a longitudinal evaluation of one of our active participants, an 85-year-old man of good health. This patient is part of a smaller cohort of Health Watch men and women who are assessed for immune function status along with the standard assessments described above. Although the patient's routine chemical and immunological parameters fall within established, healthy normal ranges, since 1984 he has experienced almost annual, prolonged (winter to spring) upper respiratory infection, demonstrated by dry coughs, sinus trouble, head and neck aches and other mild symptoms. Repeated laboratory tests and X-rays show no evidence of viral infection, and his current Health Watch profile shows nothing outside normal, healthy range values. The patient has also experienced a resurgence of allergies and other mild symptoms of progressive immune dysfunction commonly attributed to the vague processes of aging. Longitudinal balascopic evaluation of these parameters, however, pinpoints abnormal relationships among the varying, normal range parameters, providing a very different picture for the evaluation and treatment of the patient (see Table 2 and Figures 1a-1b, 2a-2b, 3a-3b, and 4).

\section{DISCUSSION}

From the patterns shown, it is clear that although this patient is maintaining healthy normal range values for chemical and immunological parameters as well as maintaining a "healthy zone" of behavioral practices, a progressive increase in abnormal relationships between these parameters is occurring. Analysis of these patterns and where new abnormalities arise helps the primary care physician to pinpoint problem areas within the myriad of healthy range values, thereby allowing for additional therapeutic approaches.

When considering those patterns shown in Figure 4, for example, it is possible to see that between the years 1986 and 1991, there is a trend towards an increasing number of immuno-metabolic abnormalities, while behavioral parameters appear to have played a much more important role in the patient's health during the earlier part of this seven year period. All of the patterns shown in this case study provide specific evidence of progressive immune dysfunction; more importantly, they provide additional clues as to where primary care intervention might be warranted and useful.

\section{CONCLUSION}

Early identification of immune dysfunction using person-specific data generated in longitudinal follow-up from patients returning for annual health status evaluation provides a basis for active intervention at all levels of preventive health care. As traditional pharmacological and less traditional psychoneuroimmunological interventions become available, balascopic analysis of trends in overall individual health status facilitates identification of otherwise healthy individuals with early immune dysfunction and may provide quantitative outcomes to monitor immune function and resistance to disease. 
Table 2. Health Watch: Health Practices Index: Patient \#15, year of Birth: 1907

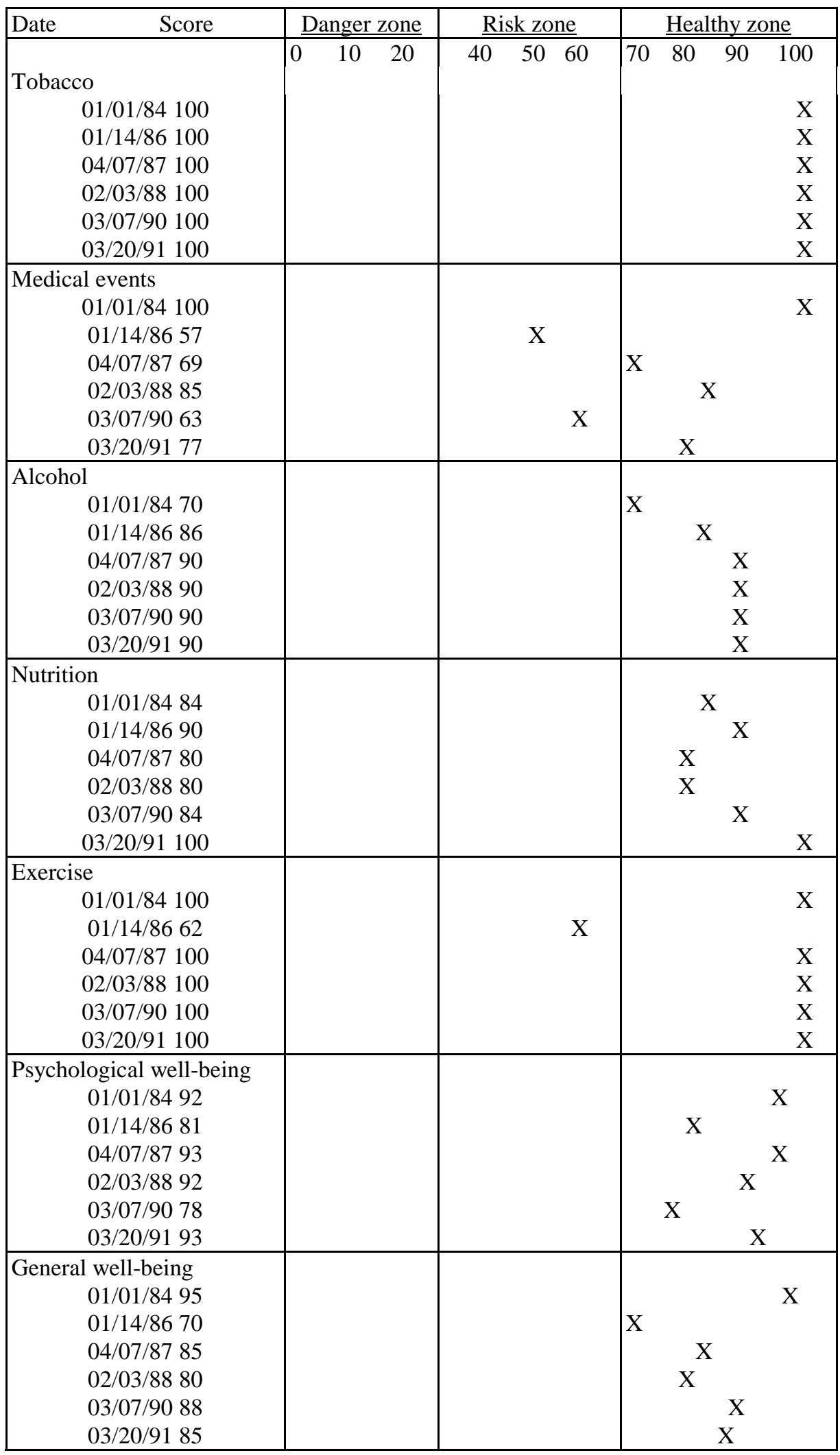




\begin{tabular}{|c|c|c|c|c|}
\hline \multicolumn{5}{|c|}{ CHEMISTRY } \\
\hline UR. & Uric Acid & 5.8 & $\mathrm{MG} / \mathrm{DL}$ & $(3.0-9.0)$ \\
\hline BUN & Urea Nitrogen & 14 & MG/DL & $(6-25)$ \\
\hline GLU & Glucose & 86 & MG/DL & $(70-110)$ \\
\hline $\mathrm{CHO}$ & Cholesterol & 221 & MG/DL & $(100-199)$ \\
\hline TRI & Triglycerides & 149 & MG/DL & $(35-200)$ \\
\hline HDL & High Density Lipoproteins & 46 & MG/DL & $(30-80)$ \\
\hline LDL & Low Density Lipoproteins & 144 & MG/DL & $(50-129)$ \\
\hline SGO & Aspartate Amino Transferase & e 41 & MU/DL & $(3-45)$ \\
\hline ALK & Alkaline Phosphatase & 94 & MU/DL & $(30-128)$ \\
\hline & IMMUNOLOGY & & & \\
\hline WBC & White Blood Cells $(x 1,000)$ & 4.9 & $\mathrm{~K} / \mathrm{UL}$ & $(3.0-10.0)$ \\
\hline BC\# & Total B Cells & 152 & \#/CUMM & $(100-600)$ \\
\hline TC\# & Total T Cells & 760 & \#/CUMM & $(800-2200)$ \\
\hline T4\# & Helper/Inducer T Cells & 501 & \#/CUMM & $(>400)$ \\
\hline $\mathrm{T} 8 \#$ & Supp./Cytotoxic T Cells & 623 & \#/CUMM & $(250-750)$ \\
\hline $4 / 8$ & T4\#/T8\# Ratio & 0.76 & Ratio & $(>0.90)$ \\
\hline & BEHAVIOR & & & \\
\hline TOB & Tobacco & 100 & & Danger Zone \\
\hline M.E & Medical Events & 100 & & $(0-33)$ \\
\hline ALH & Alcohol & 70 & & \\
\hline NUT & Nutrition & 84 & & Risk Zone \\
\hline P.A & Physical Activity/Exercise & 100 & & $(34-67)$ \\
\hline PWB & Psychological Well-being & 92 & & \\
\hline GWB & General Well-being & 95 & & $\begin{array}{c}\text { Healthy Zone } \\
(68-100)\end{array}$ \\
\hline
\end{tabular}

Figure 1a. 22 Parameters -patient \#15: 1984.

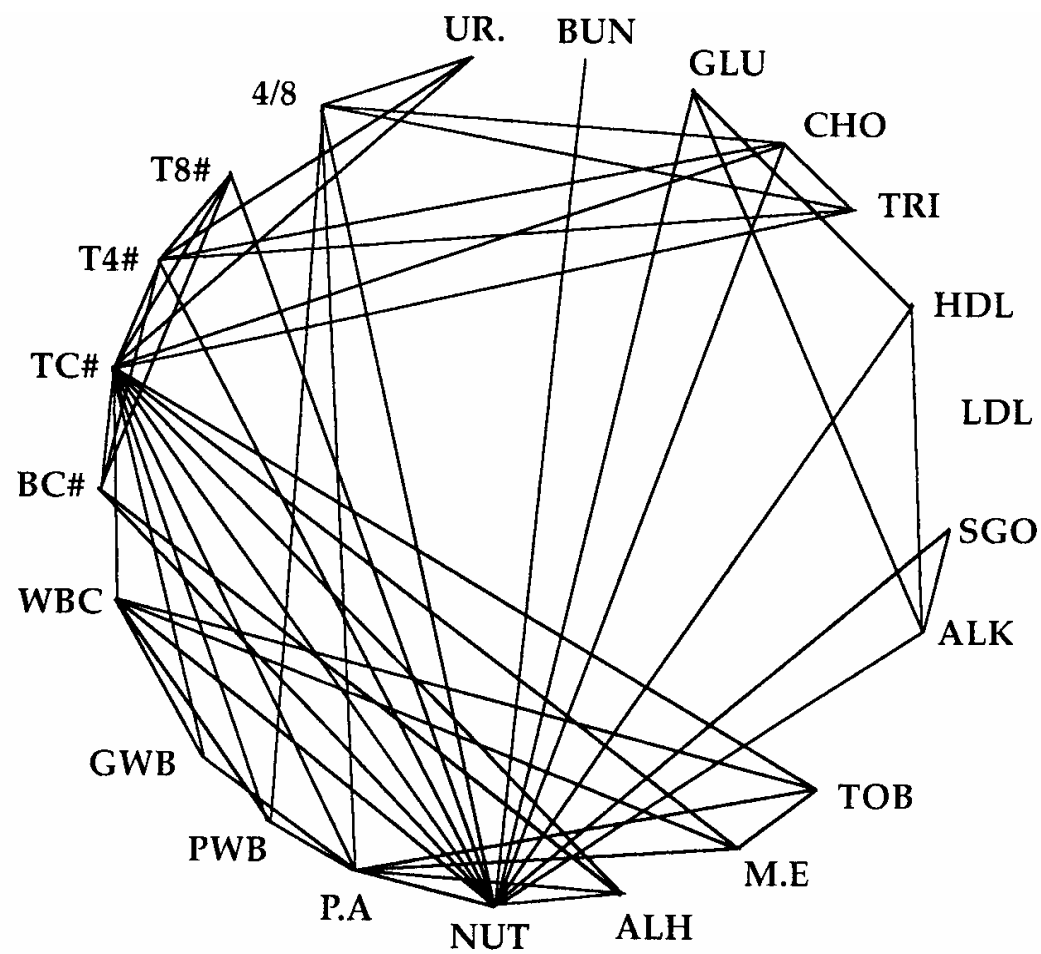

Figure 1b. Balascopic interpretation, Patient \#15: 1984. Total abnormal = 54. 


\section{CHEMISTRY}

$\begin{array}{lllll}\text { UR. } & \text { Uric Acid } & 5.8 & \text { MG/DL } & (3.0-9.0) \\ \text { BUN } & \text { Urea Nitrogen } & 13 & \text { MG/DL } & (6-25) \\ \text { GLU } & \text { Glucose } & 91 & \text { MG/DL } & (70-110) \\ \text { CHO } & \text { Cholesterol } & 212 & \text { MG/DL } & (100-199) \\ \text { TRI } & \text { Triglycerides } & 143 & \text { MG/DL } & (35-200) \\ \text { HDL } & \text { High Density Lipoproteins } & 45 & \text { MG/DL } & (30-80) \\ \text { LDL } & \text { Low Density Lipoproteins } & 139 & \text { MG/DL } & (50-129) \\ \text { SGO } & \text { Aspartate Amino Transferase 35 } & \text { MU/DL } & (3-45) \\ \text { ALK } & \text { Alkaline Phosphatase } & 99 & \text { MU/DL } & (30-128)\end{array}$

\section{IMMUNOLOGY}

$\begin{array}{lllll}\text { WBC } & \text { White Blood Cells (xl,000) } & 5.3 & \text { K/UL } & (3.0-10.0) \\ \text { BC\# } & \text { Total B Cells } & 155 & \text { \#/CUMM } & (100-600) \\ \text { TC\# } & \text { Total T Cells } & 721 & \text { \#/CUMM } & (800-2200) \\ \text { T4\# } & \text { Helper/Inducer T Cells } & 438 & \text { \#/CUMM } & (>400) \\ \text { T8\# } & \text { Supp./Cytotoxic T Cells } & 412 & \text { \#/CUMM } & (250-750) \\ \text { 4/8 } & \text { T4\#/T8\# Ratio } & 1.06 & \text { Ratio } & (>0.90)\end{array}$

\section{BEHAVIOR}

TOB Tobacco

M.E

ALH

NUT

P.A

PWB

GWB

Tobacco
Medical Events
Alcohol
Nutrition
Physical Activity/Exercise
Psychological Well-being
General Well-being

100
57
86
90
100
81
70

Figure 2a. 22 Parameters -patient \#15: 1986.

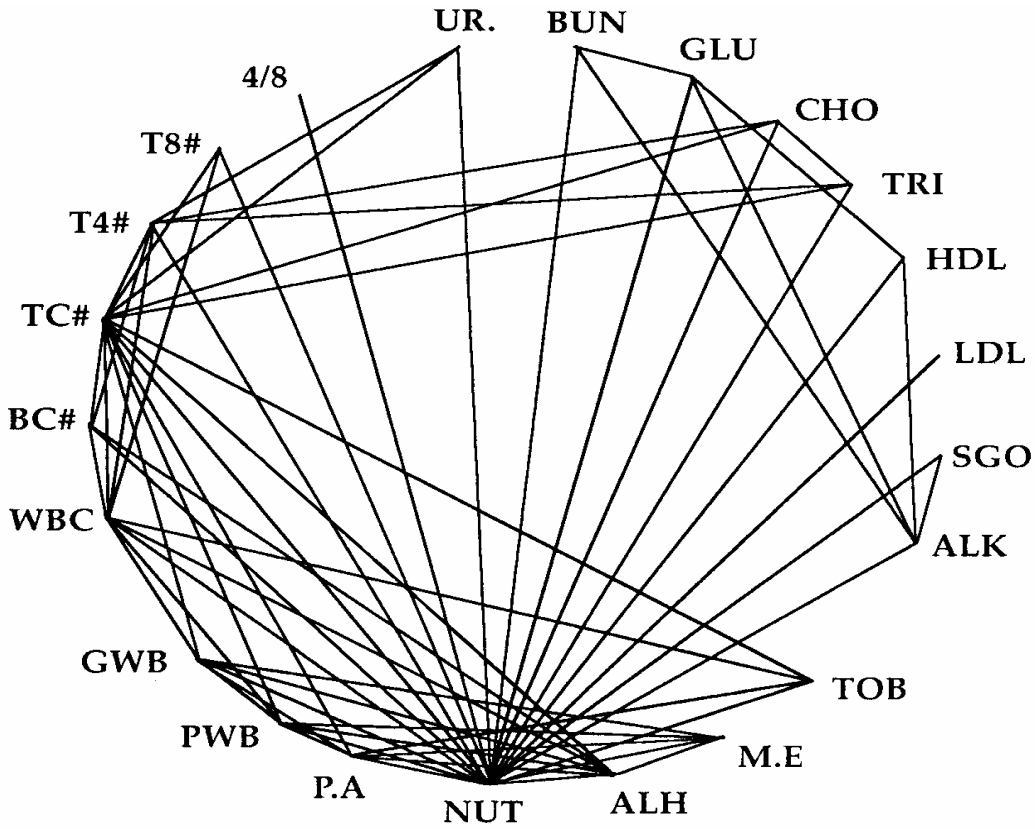

Figure 2b. Balascopic interpretation, Patient \#15: 1986. Total abnormal = 63 . 


\section{CHEMISTRY}

$\begin{array}{lllll}\text { UR. } & \text { Uric Acid } & 3.5 & \text { MG/DL } & (3.0-9.0) \\ \text { BUN } & \text { Urea Nitrogen } & 16 & \text { MG/DL } & (6-25) \\ \text { GLU } & \text { Glucose } & 88 & \text { MG/DL } & (70-110) \\ \text { CHO } & \text { Cholesterol } & 149 & \text { MG/DL } & (100-199) \\ \text { TRI } & \text { Triglycerides } & 61 & \text { MG/DL } & (35-200) \\ \text { HDL } & \text { High Density Lipoproteins } & 40 & \text { MG/DL } & (30-80) \\ \text { LDL } & \text { Low Density Lipoproteins } & 97 & \text { MG/DL } & (50-129) \\ \text { SGO } & \text { Aspartate Amino Transferase } & 24 & \text { MU/DL } & (3-45) \\ \text { ALK } & \text { Alkaline Phosphatase } & 109 & \text { MU/DL } & (30-128) \\ & & & & \\ \text { IMMUNOLOGY } & & & \\ \text { WBC } & \text { White Blood Cells (xl,000) } & 4.2 & \text { K/UL } & (3.0-10.0) \\ \text { BC\# } & \text { Total B Cells } & 228 & \text { \#/CUMM } & (100-600) \\ \text { TC\# } & \text { Total T Cells } & 888 & \text { \#/CUMM } & (800-2200) \\ \text { T4\# } & \text { Helper/Inducer T Cells } & 420 & \text { \#/CUMM } & (>400) \\ \text { T8\# } & \text { Supp./Cytotoxic T Cells } & 444 & \text { \#/CUMM } & (250-750) \\ \text { 4/8 } & \text { T4\#/T8\# Ratio } & 0.94 & \text { Ratio } & (>0.90)\end{array}$

\begin{tabular}{|c|c|c|}
\hline BEHAVIOR & & \\
\hline Tobacco & 100 & Danger Zone \\
\hline Medical Events & 77 & $(0-33)$ \\
\hline Alcohol & 90 & \\
\hline Nutrition & 100 & Risk Zone \\
\hline Physical Activity/Exercise & 100 & $(34-67)$ \\
\hline Psychological Well-being & 93 & \\
\hline General Well-being & 85 & $\begin{array}{l}\text { Healthy Zone } \\
(68-100)\end{array}$ \\
\hline
\end{tabular}

Figure 3a. 22 Parameters-patient \#15: 1991.

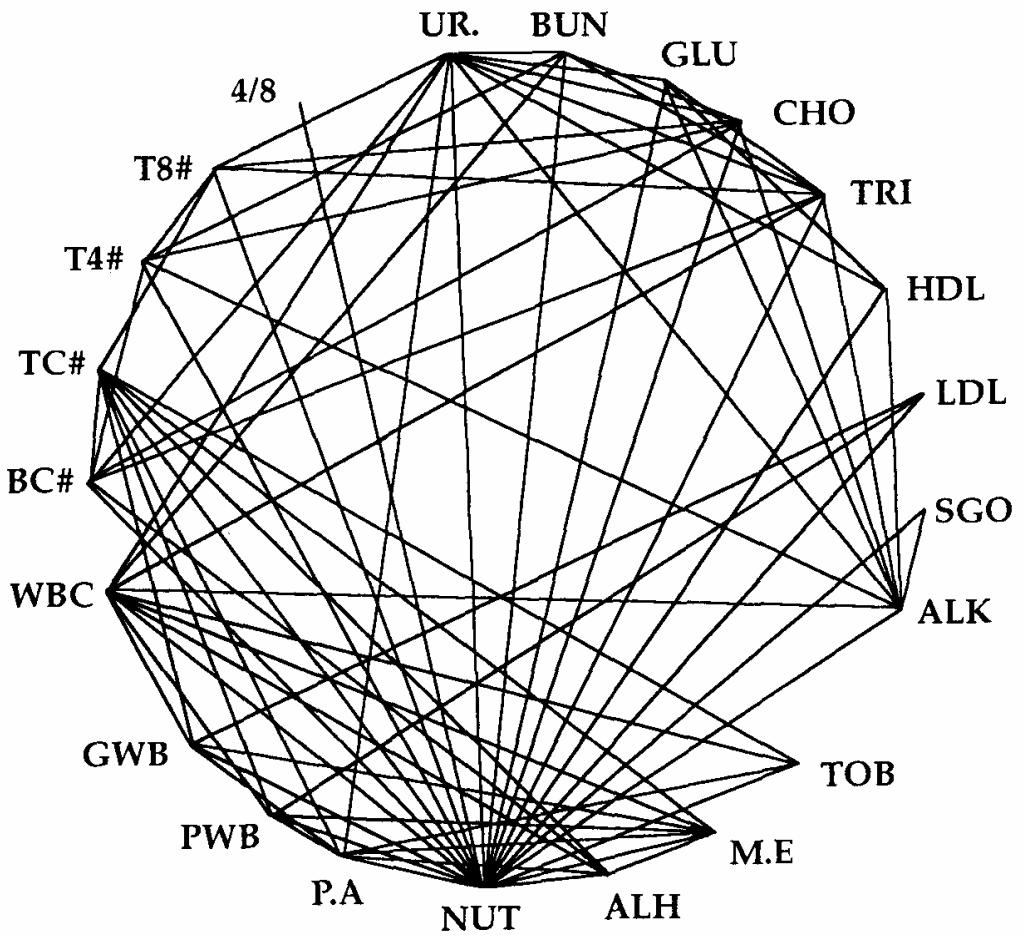

Figure 3b, Balascopic interpretation, Patient \#15: 1991. Total abnormal = 76 . 

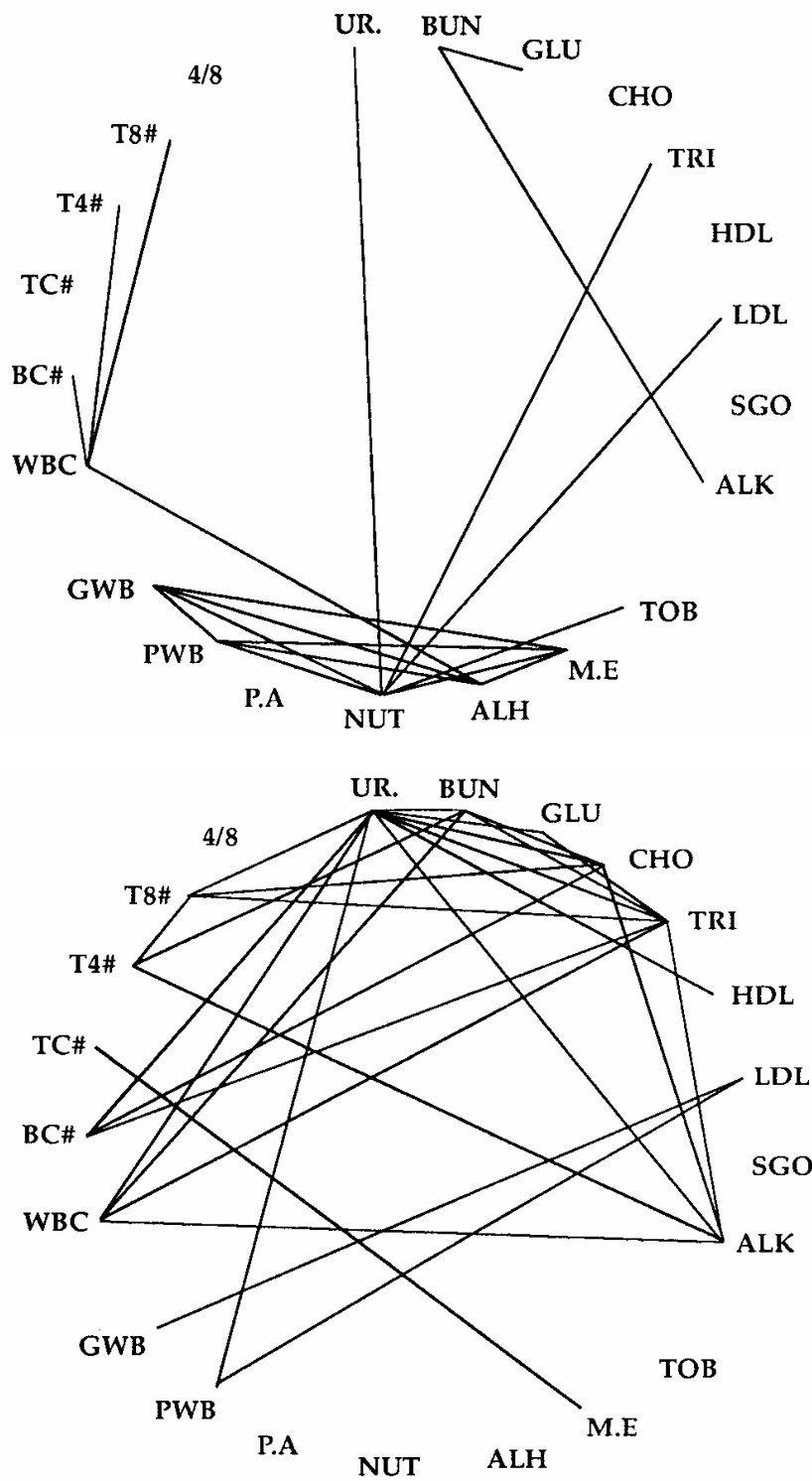

Figure 4. Balascopic patterns:

Total new abnormal relationships. Between 1984 and 1986, new abnormalities= 18; between 1986 and 1991, new abnormalities $=27$. 


\section{REFERENCES}

1. Kvitash, V., Schmidt, R.M., and Mahler, E., Computer Assisted Analysis of Behavioral, Immunological and Biochemical Data from Persons with HIV/AIDS, Health Evaluation Personal Preventive Medicine Informatics: The New Synergies, Open Joint Conference, IHEA, AMIA, and IMIA, Geneva, Switzerland, September 3-5, 1992, 28.

2. Kvitash, V., Schmidt, R.M., and Kaufman, H.S., Patterns of Hormonal-Immune-Behavioral Interactions, Proceedings of First International Conference on Hormones and Immunity, Toronto, Canada, July 4-5, 1986, 42.

3. Kvitash, V., Computerized Medical Balascopy, Proceedings of AAMSI CONGRESS 85, San Francisco, California, May 20-22, 1985, 365-369.

4. Kvitash, V., Balascopy as a Tool for Heuristic Diagnosis, Proceedings of AAMSI CONGRESS 83, San Francisco, California, $1983,121-125$.

5. Kvitash, V., Balascopy, U.S. Patent No. 4527240.

From the Center for Preventive Medicine and Health Research, California Pacific Medical Center and San Francisco State University, San Francisco, California 94115

0148-5598/93/0800-0173\$07.00/0 @ 1993 Plenum Publishing Corporation 